\section{Insertion vectors for gene therapy}

It goes without saying that efficient gene transfer is essential to gene therapy. Many different gene-transfer systems are being developed for numerous applications. Each of the envisaged applications has its own particular requirements and therefore a generally applicable, 'ideal vector' does not exist. Vector systems are usually divided into those where the vector inserts itself into the host genome and those with vectors that remain extra-chromosomal. Integrated vectors are the system of choice if persistent expression is required. However, their insertion results in an alteration of the chromosomal DNA and therefore may lead to insertional mutagenesis. Although the risk of insertional mutagenesis is a recognized disadvantage, many gene therapy protocols embrace the use of integrating vectors. Apparently, the small possibility of adverse effects of insertion mutagenesis is not seen as a major obstacle for the use of integrating vectors for clinical gene therapy.

Retroviruses are the archetype for vectors that integrate themselves into the host-cell genome. The murine retroviruses from which they are derived are notorious for their propensity to induce tumors in their natural hosts (hence their classification in the subfamily Oncovirinae). Once integrated, the provirus can perturb the expression of nearby cellular genes. In some instances, such a mutation can constitute a discrete step in the multistep process that eventually leads to cellular transformation. The unequalled efficiency with which retroviral vectors integrate their genome into the host-cell chromosomal DNA has made them the system of choice for many gene therapy applications. They have been used extensively in human gene therapy research. In more than 175 clinical trials, over 1600 patients have received retrovirus vectors. So far, not a single case has been reported in which adverse events have been attributed to insertional mutagenesis.

As murine retroviruses need both nuclear membrane degradation and active DNA synthesis in order to integrate, ${ }^{1}$ their use is limited to applications in which mitotically active cells are to be modified. The relatively short half-life of the pre-integration complex limits the window during which infection leads to provirus integration to only a few hours. In contrast to the murine retrovirus-derived vectors, lentivirus vectors are suitable for gene transfer into nonmitotic, quiescent cells, as their pre-integration complex can cross the nuclear membrane. Therefore, lentivirus vectors appear more suitable for in vivo gene transfer and major research efforts have been instigated to develop safe and effective vector systems. With the current systems, helper-free stocks of vectors which retain less than $5 \%$ of the original lentiviral genome can be generated. ${ }^{2}$

The adeno-associated virus (AAV) has also been used as a vector. AAV is a member of the Dependovirus group of the parvovirus family. This virus requires the presence of adenovirus or herpesvirus as helper, for productive infection. In the absence of helper virus, the viral genome can integrate into the cellular DNA. In the presence of the AAV-derived rep protein, the viral genome integrates preferentially at one locus on human chromosome 19. Upon subsequent infection with the herpes or adenovirus helpers, AAV can be activated. Whereas the integration of wild-type AAV has been documented in detail, the situation with AAV vectors is less clear. Although evidence suggests that at least a fraction of the AAV vectors persist as integrated copies, significant amounts of vector DNA appear to be maintained as large episomal concatemers. ${ }^{3}$

At present, the above-mentioned integrating vectors are the most widely used, but there are also ongoing efforts to generate hybrid vectors, which would be able to integrate into the genome of nonmitotic cells. Several attempts have been made to provide nonintegrating vectors (eg adenovirus vectors), which have the capacity to transduce nonmitotic cells, with integration mechanisms in order to insert the transgene into the host-cell genome. This would achieve the best of both worlds. The adenovirus-retrovirus chimeric vectors are an example of this new approach. This system consists of adenovirus vectors, which carry the gag, pol and env genes required for packaging the retroviral vector genomes, and an adenovirus vector that contains a recombinant provirus. When cells are co-infected with the above vectors, the transduced cells start to shed progeny retroviral vectors which can infect and stably modify the neighboring cells. In this way, the high titer of adenoviruses is combined with the integration capacity of retroviruses. Note that with this strategy only mitotically active cells can be stably modified.

A strategy that should be applicable to nonmitotic cells too, has recently been reported and involves the use of an AAV-adenovirus hybrid. ${ }^{4}$ In this case, one adenovirus contains a transgene flanked by sequences of the AAV inverted terminal repeat and the other carries the AAV1derived Rep78 gene. When cells are co-infected with this system, up to $35 \%$ of the clones that express the transferred resistance marker contain the transgene integrated near the hotspot of integration on chromosome 19. Intriguingly, in the latter study, the frequency of integration appears to be independent of the presence of the Rep-expressing vector, but in the absence of Rep78 the preferential integration into chromosome 19 is lost. A similar concept inspired the generation and evaluation of 
herpes simplex virus type I(HSV)/AAV hybrid vectors. ${ }^{5}$ With such vectors, transgene expression was prolonged as compared with traditional HSV-I amplicon vectors. However, in the latter study no evidence was presented confirming the integration of the hybrid vector-derived AAV cassette.

Similarly, integration or recombination systems of other origins have been exploited for integration of transgenes into the host cell genome. Such systems include various members of the transposable elements from the Tc1/mariner transposon family. ${ }^{6-8}$ In all these studies, pre-defined parts of the vector will integrate into the host cell genome. Consequently, such hybrid vectors have the risk of insertional mutagenesis in common with the more conventional integrating vectors.

The absence of integration is often used as a safety argument to promote the use of non-integrating vectors, such as adenovirus-derived vectors or plasmid vectors. This argument should be used with caution. Adenovirus DNA can recombine with chromosomal DNA, and as a result, vector sequences can become integrated into the host-cell genome. Recently, Harui and co-workers ${ }^{9}$ demonstrated that the frequency of adenovirus integration into chromosomal DNA was around $10^{-3}-10^{-5}$ events per cell. However, these frequencies have been determined in established cell lines, and it is not clear whether it is justified to extrapolate these frequencies to the in vivo situation and to estimate the risk. It would be very interesting to know the frequency with which the adenovirus vectors integrate into the host-cell genome of diploid cells. In a study with E1-containing, but replication-defective adenovirus vectors in cultures of rat diploid kidney cells, Fallaux et $a l^{10}$ reported the frequent occurrence of transformed foci. This indicates that in diploid cells too, the E1 region can become integrated into the host-cell genome as a result of illegitimate recombination, although the E1 proteins might influence the frequency of integration.

In rodents and in hamsters, adenoviruses can also become integrated into the host-cell genome, as is evidenced by the occurrence of E1- containing tumors upon injection of, for example, subgroup-A adenoviruses. If one assumes the integration frequencies in vivo to be equivalent to those observed in cell lines, then the vector amounts used for in vivo gene therapy (up to $10^{13}$ vectorparticles per dose) would imply that a significant number of cells (even millions) may acquire genome-integrated vector fragments. Thus, it is not strictly accurate to categorize adenovirus vectors as being non-integrating, when considering the risk of insertional mutagenesis. It should be noted that the insertion of vector sequences is not provoked by the adenovirus but, rather, is the result of illegitimate recombination. It seems, therefore, justified to assume that the use of other vector systems that introduce naked DNA (eg plasmids) into nuclei will also be accompanied by the integration of vector sequences into the host-cell genome.

This being the case, the use of 'non-integrating' vectors will be associated with a small but finite risk of insertional mutagenesis and perturbation of (proto-)oncogenes and tumor-suppressor genes, similar to the situation with retroviruses. However annoying this may seem at first sight, it should be seen in its proper perspective. In healthy human cells, chromosomal DNA is definitely not static and subject to mutation and recombination. Also germ line and somatic cells are modified, at a fairly high frequency, by transposable elements. ${ }^{11}$ In all, it will be extremely difficult to estimate and assess the increased risk (if any) of gene therapy for insertional mutagenesis. As long as the potential benefit outweighs the risk one should not hesitate to choose gene therapy for the treatment of severe disorders, but it is essential to ensure a proper follow-up of all patients who participate in clinical trials. In addition, great care should be taken to provide balanced views of the risks associated with the gene-transfer techniques and to abstain from sweeping generalizations. If given proper counseling, the people involved can make their judgments based on their personal perception of the risk and benefits. We should realize that we are making such judgments every day. Don't most of us expose ourselves to sunlight to acquire a tan, at the cost of an increased risk of skin cancer due to UVinduced mutagenesis? ${ }^{12}$

FHE Schagen, HJ Rademaker, FJ Fallaux, RC Hoeben Department of Molecular Cell Biology Leiden University Medical Center, PO Box 9503 2300 RA Leiden, The Netherlands

\section{References}

1 Miller DG, Adam MA, Miller AD. Gene transfer by retrovirus vectors occurs only in cells that are actively replicating at the time of infection. Mol Cell Biol 1990; 10: 4239-4242.

2 Amado RG, Chen IS. Lentiviral vectors - the promise of gene therapy within reach? Science 1999; 285: 674-676.

3 Yang J et al. Concatamerization of adeno-associated virus circular genomes occurs through intermolecular recombination. J Virol 1999; 73: 9468-9477.

4 Recchia A et al. Site-specific integration mediated by a hybrid adenovirus/adeno-associated virus vector. Proc Natl Acad Sci USA 1999; 96: 2615-2620.

5 Fraefel C et al. Gene transfer into hepatocytes mediated by helper virus-free HSV/AAV hybrid vectors. Mol Med 1997; 3: 813-825.

6 Zhang L et al. The Himar1 mariner transposase cloned in a recombinant adenovirus vector is functional in mammalian cells. Nucleic Acids Res 1998; 26: 3687-3693.

7 Schouten GJ et al. Transposon Tc1 of the nematode Caenorhabditis elegans jumps in human cells. Nucleic Acids Res 1998; 26: 3013-3017.

8 Ivics Z, Hackett PB, Plasterk RH, Izsvak Z. Molecular reconstruction of Sleeping Beauty, a Tc1-like transposon from fish, and its transposition in human cells. Cell 1997; 91: 501-510.

9 Harui A, Suzuki S, Kochanek S, Mitani K. Frequency and stability of chromosomal integration of adenovirus vectors. J Virol 1999; 73: 6141-6146.

10 Fallaux FJ, van der Eb AJ, Hoeben RC. Who's afraid of replication-competent adenoviruses? Gene Therapy 1999; 6: 709-712.

11 Kazazian HH Jr. An estimated frequency of endogenous insertional mutations in humans. Nat Genet 1999; 22: 130.

12 Jonason AS et al. Frequent clones of p53-mutated keratinocytes in normal human skin. Proc Natl Acad Sci USA 1996; 93: 14025-14029. 